\title{
Conceptualizations of E-recruitment: A Literature Review and Analysis
}

\author{
Mike Abia ${ }^{1(\bowtie)}$ and Irwin Brown ${ }^{2(凶)}$ \\ ${ }^{1}$ Department of Computer Science, Namibia University \\ of Science and Technology, 13 Jackson Kaujeua Street, Windhoek, Namibia \\ abiamike@gmail.com \\ ${ }^{2}$ Department of Information Systems, University of Cape Town, Rondebosch, \\ Cape Town 7701, South Africa \\ Irwin.Brown@uct.ac.za
}

\begin{abstract}
There is diversity in understanding of electronic recruitment (e-recruitment) which results in confusion on the meaning and use of the term. The purpose of this paper is to bring conceptual clarity by investigating the alternative conceptualizations of e-recruitment in academic literature. Using Grounded Theory Methodology (GTM) techniques we analyzed literature to reveal five alternative conceptualizations; these being: (1) E-recruitment as a Technology Tool, (2) E-recruitment as a System, (3) E-recruitment as a Process, (4) E-recruitment as a Service, and (5) E-recruitment as a Proxy. The conceptualizations map to the scope of the definition and utilization of e-recruitment. Identifying conceptualizations of e-recruitment sets a platform for further research. Further research may include determining the relationships between the conceptualizations and determining conceptualizations in different settings among many other possible research focus topics.
\end{abstract}

Keywords: E-recruitment $\cdot$ Conceptualization $\cdot$ Literature review $\cdot$ Grounded Theory Methodology

\section{Introduction}

E-recruitment has many labels that include; internet recruitment, online recruitment, web-recruitment and many others. Unlike traditional recruitment, e-recruitment makes use of information technology to handle the recruitment processes. Breaugh et al. [1] defined a recruitment model that presents the recruitment process at a macro level with the following activities: Setting recruitment objectives, developing a strategy, performing the recruitment activity and obtaining and evaluating recruitment results. Recruiters compete with each other for candidates (jobseekers suitable for available jobs), while jobseekers compete for jobs; which drives both groups to adopt information technologies at accelerated rates in order to take the strain out of some of the recruitment activities [2-7]. "For most job seekers, the Internet is where the action is" 
[3, p. 140]. Thus, to get candidates, recruiters need to move swiftly to locate and hire, which may require use of a multitude of information technologies in the process [8, p. 130].

There is evidence in research papers that academic disciplines and stakeholders have varied definitions of e-recruitment. The variety of definitions of e-recruitment is expected because it is part of e-HRM (electronic Human Resource Management) that has in itself different definitions depending on the context [6, p. 26], [9, p. 98]. Studies based on these definitions tend to reveal overlapping and contradictory results due to the overlaps or differences in definitions [9, p. 100]. The differences in definitions, aside from being problematic, is evidence of the variety in conceptualization of erecruitment. Thus to find a standard definition of e-recruitment, conceptualizations of erecruitment need to be known. To our knowledge, no research paper in e-recruitment has focused on conceptualization of e-recruitment, however there are studies in other areas of information systems (IS) that have focused on conceptualization [10-19]. Most view conceptualization as the formulation of a view about the nature of a phenomenon. The research questions to be answered are:

1. What conceptualizations of e-recruitment exist in literature?

2. How can the conceptualizations be described and explained?

\section{Methodology for Reviewing Literature}

Because of the large number of research papers on e-recruitment we aimed at selecting papers for review that would embrace the full variety of conceptualizations of erecruitment. Also, we wanted a flexible review methodology that would allow for selection and analysis of papers simultaneously, as the conceptualizations emerged, rather than a sequential review methodology that required all research papers to be selected beforehand. Such flexibility is provided for by applying grounded theory methodology (GTM) as a suitable review methodology [20]. GTM techniques used in this study included open coding to identify concepts, constant comparative analysis to refine and differentiate conceptualizations, and theoretical sampling to identify further relevant literature [21, 22].

Figure 1 is a flowchart depicting how the literature was processed from search until conceptualizations of e-recruitment were identified, saturated and completed. 
Key to diagram on GTM for reviewing literature

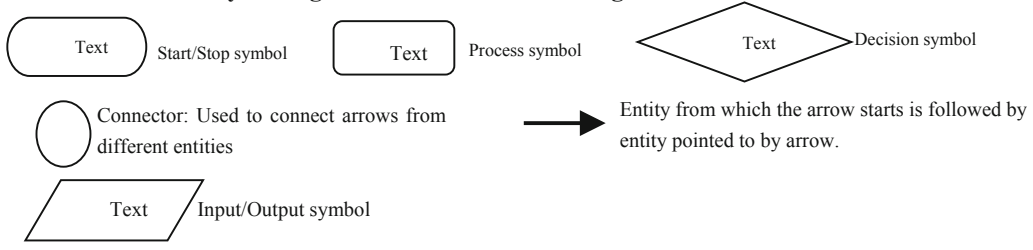

Flowchart of the GTM process

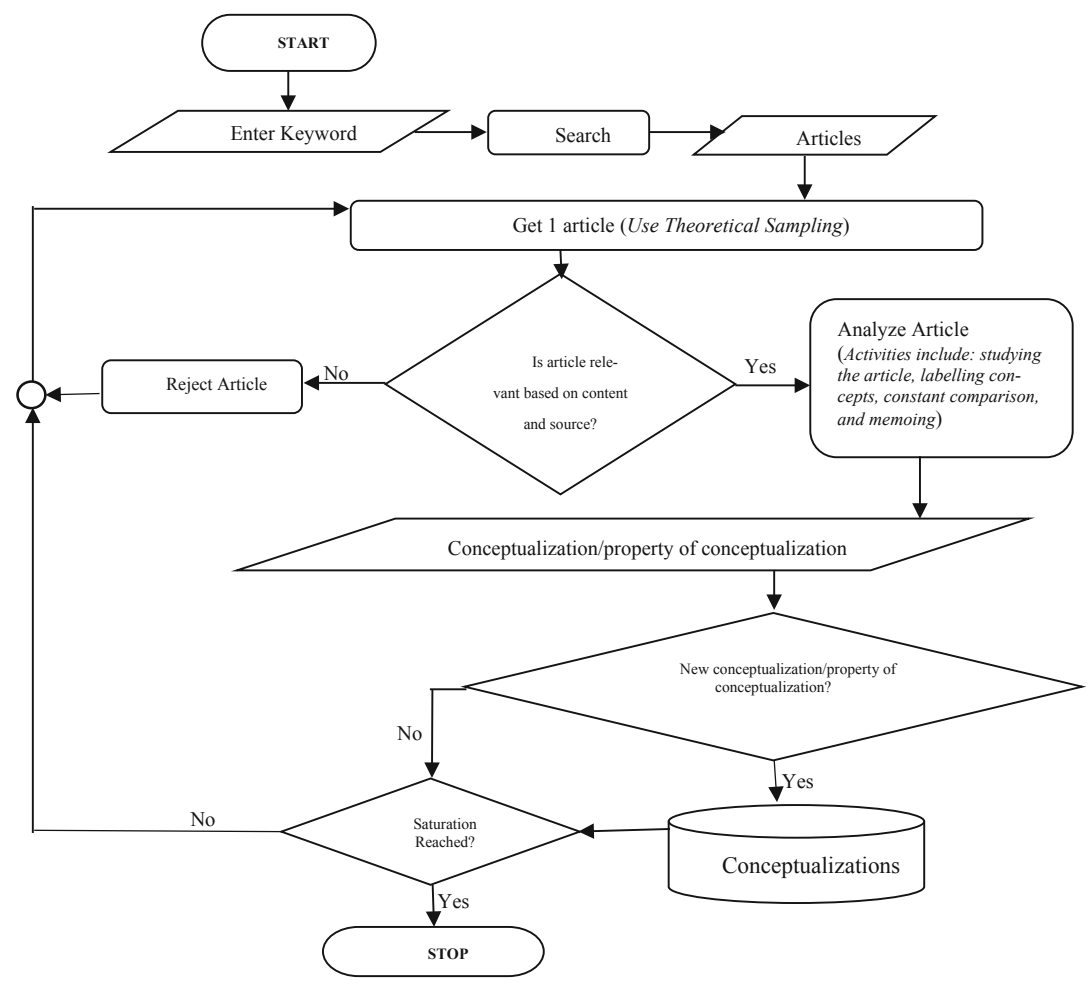

Fig. 1. GTM for reviewing literature

\subsection{Searching for Articles}

We used the web search engine Google Scholar to search electronically for the articles. We fed keywords synonymic with the word e-recruitment into the searching tool. These are: e-recruiting, e-HRM, e-Human Resource Management, electronic HRM, electronic Human Resource Management, e-recruiting, e-recruitment, internet recruiting, internet recruitment, online recruiting, online recruitment, recruiting online, recruiting on the internet, recruiting on the web, recruitment online, web-based recruiting, web-based recruitment, web recruiting, web recruitment [20]. 
After an initial search on Google Scholar and filtering of articles for relevancy based on paper titles and abstracts we had 445 journal articles and conference papers published in the period 1998 to 2019 in approximately 145 sources. The search process provided a set of many articles, but it did not qualify all of them as useful for the review. The selection process had to take place to sample useful and relevant articles for the review.

\subsection{Theoretical Sampling of Articles}

Ideally all papers on e-recruitment needed to be included in the review. Alternatively, papers included in the analysis had to be a representative sample of all papers in erecruitment that were relevant for the developing conceptualizations. However with the vast amount of research in e-recruitment and the huge number of articles from our search and filtering it would be difficult or time consuming to include all relevant erecruitment research articles for the review. The alternative of having a representative sample was viable and using GTM's theoretical sampling [21] was feasible for the objectives of this research to be met.

An initial article to be analyzed was picked from the population of 445 articles. Picking of subsequent articles for inclusion in the sample was informed by the emerging conceptualizations. Theoretical sampling was performed until all the conceptualizations got saturated and completed. Glaser [22] defines saturation as a state where new data does not bring new properties to the concepts. In an effort to attain completeness a check was done to make sure all conceptualizations were included. Theoretical sampling ended when saturation and completeness was achieved. This is the point at which the number of research articles involved in identifying conceptualizations in e-recruitment were counted. In the end 26 research articles were relevant for identifying and explaining conceptualizations of e-recruitment.

\subsection{Analyzing Articles}

Analysis of the articles that let conceptualizations of e-recruitment emerge (see Fig. 1) required that constant comparison be applied by comparing codes to codes and concepts to concepts to find and note their relationships and further develop the labelled conceptualizations [21, 22]. The emerging conceptualizations served as a framework for further selection of articles and using systematic deduction from the emerging conceptualization possibilities and probabilities were determined to guide the next cycle of article selection. Memos were created to note the emergent ideas. Memoed ideas also served to direct which article to sample next.

Every sampled article was investigated for its perspective on the essence of erecruitment or the most essential or most vital part that embodied the conceptualization of e-recruitment. Indicators in the article brought forth the conceptualizations. The moment of departure from the analysis to getting another article for analysis came only after the article was fully analyzed. The resulting conceptualizations are detailed in the next section. 


\section{Conceptualizations of E-recruitment}

Five conceptualizations of e-recruitment emerged from extent literature, namely: erecruitment as a technology tool, e-recruitment as a system, e-recruitment as a process, e-recruitment as a service and e-recruitment as a proxy. Although many of the articles had a mixture of conceptualizations, one or two stood out in each article and for each conceptualization Table 1 gives example research articles. After the presentation in Table 1 each of the conceptualizations is described and explained in sub-sections that follow.

Table 1. Conceptualizations of E-recruitment

\begin{tabular}{|c|c|c|}
\hline $\begin{array}{l}\text { Conceptualization of e-Recruitment } \\
\text { (Label Created in this Study) }\end{array}$ & Description & Articles \\
\hline $\begin{array}{l}\text { 1. E-recruitment as a Technology } \\
\text { Tool }\end{array}$ & $\begin{array}{l}\text { E-recruitment is viewed in some studies as } \\
\text { a technology tool }\end{array}$ & {$[23]$} \\
\hline 2. E-recruitment as a System & $\begin{array}{l}\text { E-recruitment is a group of independent but } \\
\text { interrelated elements comprising a unified } \\
\text { whole. These elements include technology, } \\
\text { society, organizations, etc. }\end{array}$ & [23-29] \\
\hline 3. E-recruitment as a Process & $\begin{array}{l}\text { E-recruitment is a set of systematic well- } \\
\text { coordinated activities. The activities are } \\
\text { done by information technology or } \\
\text { traditionally }\end{array}$ & {$[25,29-37]$} \\
\hline $\begin{array}{l}\text { 4. E-recruitment as a Service } \\
\text { a. E-recruitment as a Repository } \\
\text { b. E-recruitment as a Medium } \\
\text { c. E-recruitment as a Program } \\
\text { (E-recruitment as an } \\
\text { Implemented Algorithm) }\end{array}$ & $\begin{array}{l}\text { E-recruitment is a service to recruitment. It } \\
\text { cannot be entrusted to do all that is needed } \\
\text { for successful recruitment, therefore it only } \\
\text { provides certain functionalities } \\
\text { a. E-recruitment provides storage facilities } \\
\text { for recruitment data } \\
\text { b. E-recruitment is a communications } \\
\text { conduit between stakeholders in } \\
\text { recruitment } \\
\text { c. E-recruitment is a set of precise rules for } \\
\text { solving a problem }\end{array}$ & $\begin{array}{l}{[7,38,39]} \\
\text { a. }[33,37, \\
40] \\
\text { b. }[31,41- \\
44] \\
\text { c. }[23,25, \\
35]\end{array}$ \\
\hline 5. E-recruitment as a Proxy & $\begin{array}{l}\text { E-recruitment acts on behalf of } \\
\text { organizational and societal entities }\end{array}$ & {$[35,45,46]$} \\
\hline
\end{tabular}

\subsection{E-recruitment as a Technology Tool}

E-recruitment as a technology tool is a conceptualization of e-recruitment as a technical artefact [19]. This means is demonstrated by Faliagka et al. [23] who presented a tool to automate the ranking of applicants in recruitment. 


\subsection{E-recruitment as a System}

Studies that view e-recruitment as a system conceptually divide e-recruitment into independent but interrelated elements, at the core of which is information technology, society, organizations, etc. The system view allows each component to receive input from the other elements and produce input for other components [25]. The system view of e-recruitment assigns all automating functions to the IT artefact of the system while organizational recruitment experts evaluate the outcome [24]. While some stakeholders view e-recruitment as a system, others view it as a process.

\subsection{E-recruitment as a Process}

Instead of focusing on entities, the process view of e-recruitment focuses on erecruitment activities [37]. There is no attempt to set boundaries between the IT artefact, society and organization, but activities are clearly identified and can be performed by either the IT artefact or by human actors. Examples include e-recruitment being seen as data collection activity using an online system [33]. However recruitment activities can be performed by human actors too [37]. With the process view of erecruitment the end goal is the execution of all the recruitment activities.

\subsection{E-recruitment as a Service}

The view exists that e-recruitment is a service to recruiters and job-seekers. Many erecruitment platforms are independent of the organizations or societies they serve. Subviews of e-recruitment as a service include: e-recruitment as a repository, e-recruitment as a medium, and e-recruitment as a program.

E-recruitment as a repository. Some studies portrayed e-recruitment as a repository for data about jobs, recruiters and employers [40]. In another study online forms were filled in by jobseekers and the data provided on the forms was stored for recruiters and other stakeholders to retrieve [33]. While the view of e-recruitment as a repository is usually held when e-recruitment is newly adopted, other services follow suit.

E-recruitment as a medium is another view held, e.g. Bartram [41] portrays erecruitment as a facilitator of communication between jobseekers and organizations. Traditional media like newspaper [42] are sometimes found inconvenient thus erecruitment takes their place. Some organizations employ e-recruiters who form part of e-recruitment and serve to link the IT artefact and other elements in recruitment. Although e-recruitment as a medium improves communication speed it also comes with a downside, e.g. information overload [37].

E-recruitment as a program is a view that associates e-recruitment with calculations and logical interpretation and processing of data. One study included, as an algorithmic module, a Pre-screening Management System to automatically assess the extent of match between an applicant's qualification and job requirements [25]. Such module or similar modules are found in many e-recruitment systems given the high volumes of applications associated with e-recruitment. Therefore, many studies espouse the view that e-recruitment serves to provide a convenient matching program. 


\subsection{E-Recruitment as a Proxy}

Orlikowski and Iacono [19] reveal the pervasiveness of the proxy view of the IT artefact in IS literature. E-recruitment may act to present the image of the company, culture of the company, etc. Braddy et al. [45] examined the effects of website content features on people's perceptions of organizational culture. Their study implies that erecruitment, especially the IT artefact (website) acts on behalf of some corporate image management entity in the organization. Some studies focused on website content [45], while others focused on website characteristics [46].

\section{Contribution and Implications of Conceptualizations of E-recruitment}

Conceptualizations of e-recruitment contribute to understanding of e-recruitment and have implications for both practice and research as discussed in this section.

\subsection{Contribution of the Research}

This study mapped the scope of the definition of e-recruitment by explaining the diversity in understanding. This mapping was done by identifying five conceptualizations of erecruitment and labelling them as: E-recruitment as a Technology Tool, E-recruitment as a System, E-recruitment as a Process, E-recruitment as a Service and E-recruitment as a Proxy. Taking note of conceptualizations provides practitioners with a tool to enhance productivity while allowing researchers to have more focus in their research.

\subsection{Implications of Conceptualizations of E-recruitment}

The implications of conceptualizations of e-recruitment stem from being able to attach a label to the said stakeholders' conceptualizations and put it to their trade or scholarly pursuits. Labelling conceptualizations provides a pathway to standardization of e-recruitment. The benefits of such standardization include having common understanding of concepts, and ease of communication. While these are overarching implications, some implications are specific to practice or research.

Implications for Practice. Labelled and well defined conceptualizations of e-recruitment sets bounds on what practitioners should expect in their practice and strive towards when they adopt a particular conceptualization. Well defined conceptualizations as ones in this study provide alternative conceptualization options that practitioners can adopt depending on their needs. Practitioners can always adopt a conceptualization that best reflects their situation. As there are implications for practice, there are implications for research as well.

Implications for Research. Through this identification, description and explanation of conceptualizations of e-recruitment, there are a number of conceptualizations to consider. Therefore, focus on a specific conceptualization or focus on specific conceptualizations is possible. Such focus allows the researcher to delimit research. 


\section{Conclusion and Further Research}

\subsection{Conclusion}

The study highlighted the problem of diversity in understanding of e-recruitment that goes without explicit attention in literature and proposed that identifying and labelling the varied conceptualizations of e-recruitment can be part of better articulation of the diversity. Using GTM, literature on e-recruitment was reviewed and conceptualizations of e-recruitment were identified. Taking note of conceptualizations provides practitioners with a tool to enhance productivity while allowing researchers to have more focus in their research. In addition this study provides insight into directions for potential further study.

\subsection{Further Research}

While this research contributes to understanding of e-recruitment, further research related to it can respond to several issues which are not addressed herein. Understanding of relationships between conceptualizations helps to avoid conceptual chaos. Therefore, further research aimed at relating the conceptualizations is essential. Conceptualizations of e-recruitment may be compared to conceptualizations of other forms of e-phenomena, and hence to the development of more general understanding of IS and the IT artefact.

\section{References}

1. Breaugh, J.A., Macan, T.H., Grambow, D.M.: Employee recruitment: current knowledge and directions for future research. Int. Rev. Ind. Organ. Psychol. 23, 45-82 (2008)

2. Borstorff, P.C., Marker, M.B., Bennett, D.S.: Online recruitment: attitudes and behaviors of job seekers. J. Strateg. E-Commer. 5(1/2), 1 (2007)

3. Cappelli, P.: Making the most of on-line recruiting. Harv. Bus. Rev. 79(3), 139-146 (2001)

4. Cober, R.T., Brown, D.J., Levy, P.E.: Form, content, and function: an evaluative methodology for corporate employment web sites. Hum. Resour. Manage. 43(2-3), 201218 (2004)

5. Pfieffelmann, B., Wagner, S.H., Libkuman, T.: Recruiting on corporate web sites: perceptions of fit and attraction. Int. J. Sel. Assess. 18(1), 40-47 (2010)

6. Simón, C., Esteves, J.: The limits of institutional isomorphism in the design of e-recruitment websites: a comparative analysis of the USA and Spain. Int. J. Hum. Resour. Manag. 27(1), 23-44 (2016)

7. Smyth, B., Bradley, K., Rafter, R.: Personalization techniques for online recruitment services. Commun. ACM 45(5), 39-40 (2002)

8. Koong, K.S., Liu, L.C., Williams, D.L.: An identification of internet job board attributes. Hum. Syst. Manag. 21(2), 129-135 (2002)

9. Bondarouk, T., Parry, E., Furtmueller, E.: Electronic HRM: four decades of research on adoption and consequences. Int. J. Hum. Resour. Manag. 28(1), 98-131 (2017) 
10. Doherty, N.F., Coombs, C.R., Loan-Clarke, J.: A re-conceptualization of the interpretive flexibility of information technologies: redressing the balance between the social and the technical. Eur. J. Inf. Syst. 15(6), 569-582 (2006). https://doi.org/10.1057/palgrave.ejis. 3000653

11. Pinsonneault, A., Heppel, N.: Anonymity in group support systems research: a new conceptualization, measure, and contingency framework. J. Manag. Inf. Syst. 14(3), 89-108 (1997). https://doi.org/10.1080/07421222.1997.11518176

12. Sun, J., Teng, J.T.C.: Information systems use: construct conceptualization and scale development. Comput. Hum. Behav. 28(5), 1564-1574 (2012). https://doi.org/10.1016/j. chb.2012.03.016

13. Hong, W., Thong, J.Y.L.: Internet privacy concerns: an integrated conceptualization and four empirical studies. MIS Q. 37(1), 275-298 (2013)

14. George, J.F.: The conceptualization and development of organizational decision support systems. J. Manag. Inf. Syst. 8(3), 109-125 (1991)

15. Wang, Y.-S., Liao, Y.-W.: The conceptualization and measurement of m-commerce user satisfaction. Comput. Hum. Behav. 23(1), 381-398 (2007). https://doi.org/10.1016/j.chb. 2004.10.017

16. Tilly, R., Posegga, O., Fischbach, K., Schoder, D.: Towards a conceptualization of data and information quality in social information systems. Bus. Inf. Syst. Eng. 59(1), 3-21 (2017). https://doi.org/10.1007/s12599-016-0459-8

17. Jasperson, J., Carter, P.E., Zmud, R.: A comprehensive conceptualization of post-adoptive behaviors associated with information technology enabled work systems. MIS Q. 29(3), 525 (2005). https://doi.org/10.2307/25148694

18. Barki, H., Titah, R., Boffo, C.: Information system use-related activity: an expanded behavioral conceptualization of individual-level information system use. Inf. Syst. Res. 18 (2), 173-192 (2007)

19. Orlikowski, W.J., Iacono, C.S.: Research commentary: desperately seeking the 'IT' in IT research - a call to theorizing the IT artifact. Inf. Syst. Res. 12(2), 121-134 (2001)

20. Wolfswinkel, J., Furtmueller, E., Wilderom, C.: Using grounded theory as a method for rigorously reviewing literature. Eur. J. Inf. Syst. 22(1), 45-55 (2013)

21. Glaser, B.G.: Advances in the Methodology of Grounded Theory: Theoretical Sensitivity. Sociology Press, California (1978)

22. Glaser, B.G.: Doing Grounded Theory: Issues and Discussions. Sociology Press, California (1998)

23. Faliagka, E., Tsakalidis, A., Tzimas, G.: An integrated e-recruitment system for automated personality mining and applicant ranking. Internet Res. Bradf. 22(5), 551-568 (2012)

24. Chiwara, J.R., Chinyamurindi, W.T., Mjoli, T.Q.: Factors that influence the use of the internet for job-seeking purposes amongst a sample of final-year students in the Eastern Cape Province of South Africa. SA J. Hum. Resour. Manag. 15(1), 1-9 (2017)

25. Lee, I.: An architecture for a next-generation holistic e-recruiting system. Commun. ACM 50 (7), 81-85 (2007)

26. Yoon Kin Tong, D.: A study of e-recruitment technology adoption in Malaysia. Ind. Manag. Data Syst. 109(2), 281-300 (2009)

27. Braddy, P., Thompson, L.F., Wuensch, K., Grossnickle, W.: Internet recruiting: the effects of web page design features. Soc. Sci. Comput. Rev. 21(3), 374-385 (2003)

28. Pavon, F., Brown, I.: Factors influencing the adoption of the world wide web for job-seeking in South Africa. South Afr. J. Inf. Manag. 12(1), 1-9 (2010)

29. Lee, I.: Modeling the benefit of e-recruiting process integration. Decis. Support Syst. 51(1), 230-239 (2011) 
30. Ehrhart, K.H., Mayer, D.M., Ziegert, J.C.: Web-based recruitment in the millennial generation: work-life balance, website usability, and organizational attraction. Eur. J. Work Organ. Psychol. 21(6), 850-874 (2012)

31. Feldman, D.C., Klaas, B.S.: Internet job hunting: a field study of applicant experiences with on-line recruiting. Hum. Resour. Manag. 41(2), 175-192 (2002)

32. Parry, E., Tyson, S.: An analysis of the use and success of online recruitment methods in the UK. Hum. Resour. Manag. J. 18(3), 257-274 (2008)

33. García-Izquierdo, A.L., Aguinis, H., Ramos-Villagrasa, P.J.: Science-practice gap in erecruitment. Int. J. Sel. Assess. 18(4), 432-438 (2010)

34. Kashi, K., Zheng, C.: Extending technology acceptance model to the e-recruitment context in Iran. Int. J. Sel. Assess. 21(1), 121-129 (2013)

35. Jansen, B.J., Jansen, K.J., Spink, A.: Using the web to look for work: implications for online job seeking and recruiting. Internet Res. 15(1), 49-66 (2005)

36. Smith, A.D., Rupp, W.T.: Managerial challenges of e-recruiting: extending the life cycle of new economy employees. Online Inf. Rev. 28(1), 61-74 (2004)

37. Llorens, J.J.: A model of public sector e-recruitment adoption in a time of hyper technological change. Rev. Public Pers. Adm. 31(4), 410-423 (2011)

38. Koch, T., Gerber, C., de Klerk, J.J.: The impact of social media on recruitment: are you LinkedIn? SA J. Hum. Resour. Manag. 16(2), 1-14 (2018)

39. Vidros, S., Kolias, C., Kambourakis, G.: Online recruitment services: another playground for fraudsters. Comput. Fraud Secur. 2016(3), 8-13 (2016)

40. Wang, B., Guo, X.: Online recruitment information as an indicator to appraise enterprise performance. Online Inf. Rev. Bradf. 36(6), 903-918 (2012)

41. Bartram, D.: Internet recruitment and selection: kissing frogs to find princes. Int. J. Sel. Assess. 8(4), 261-274 (2000)

42. Selden, S., Orenstein, J.: Government e-recruiting web sites: the influence of e-recruitment content and usability on recruiting and hiring outcomes in US state governments. Int. J. Sel. Assess. 19(1), 31-40 (2011)

43. Sylva, H., Mol, S.T.: E-recruitment: a study into applicant perceptions of an online application system. Int. J. Sel. Assess. 17(3), 311-323 (2009)

44. Van Hoye, G., Lievens, F.: Investigating web-based recruitment sources: employee testimonials vs word-of-mouse. Int. J. Sel. Assess. 15(4), 372-382 (2007)

45. Braddy, P., Meade, A., Michael, J., Fleenor, J.: Internet recruiting: effects of website content features on viewers' perceptions of organizational culture. Int. J. Sel. Assess. 17(1), 19-34 (2009)

46. Walker, H.J., Feild, H.S., Giles, W.F., Bernerth, J.B., Short, J.C.: So what do you think of the organization? A contextual priming explanation for recruitment web site characteristics as antecedents of job seekers' organizational image perceptions. Organ. Behav. Hum. Decis. Process. 114(2), 165-178 (2011) 